\title{
Gastric Banding Causing Adnexal Entrapment During Pregnancy
}

\author{
Zvi Vaknin, MD, Hasan Kais, MD, Noga Fuchs, MD, Sharon Berger, MD, Moty Pansky, MD, \\ Noam Smorgick, MD, Msc \\ Department of Obstetrics and Gynecology, Assaf Harofe Medical Center, affiliated with the Sackler Faculty of Medicine, \\ Tel Aviv University, Beer Yaakov, Israel (Drs. Vaknin, Fuchs, Berger, Pansky; Smorgick). \\ Department of General Surgery B, Assaf Harofe Medical Center, affiliated with the Sackler Faculty of Medicine, Tel Aviv \\ University, Beer Yaakov, Israel (Dr. Kais).
}

\begin{abstract}
Introduction: Laparoscopic adjustable gastric banding (LAGB) is commonly used for the management of morbid obesity in women of reproductive age. This surgery was found to be safe and well tolerated during pregnancy, with a lower incidence of gestational diabetes and maternal hypertension than was found in control subjects. The most common complications of LABG include gastric perforation, band slippage, and port disconnection. We present an unusual complication of LABG during pregnancy.

Case Description: The patient is a 34-year-old pregnant woman who underwent LABG 3 years prior to her pregnancy and presented at 14 weeks' gestation with acute left lower quadrant abdominal pain. Laparoscopy was performed for suspected adnexal torsion (based on clinical presentation and sonographic findings), revealing an entrapment of the left adnexum by the connecting tube of the LAGB device. The left adnexum was released with no residual complications.
\end{abstract}

Discussion: This complication, although rare, should be considered during early pregnancy.

Key Words: Laparoscopic adjustable gastric banding, Pregnancy, adnexal torsion.

Citation Vaknin Z, Kais H, Fuchs N, Berger S, Pansky M, Smorgick N. Gastric banding causing adnexal entrapment during pregnancy. CRSLS e2014.00216. DOI 10.4293/CRSLS.2014.00216.

Copyright (C) 2014 SLS This is an open-access article distributed under the terms of the Creative Commons Attribution-Noncommercial-ShareAlike 3.0 Unported license, which permits unrestricted noncommercial use, distribution, and reproduction in any medium, provided the original author and source are credited.

Address correspondence to: Noam Smorgick, MD, Department of Obstetrics and Gynecology, Assaf Harofe Medical Center, Zerifin, Israel, 70300, Tel: +972-8-9779000, Fax: +972-8-9778026. E-mail: noam_yossi@yahoo.com

\section{INTRODUCTION}

Bariatric surgeries are gaining popularity as the leading treatment for morbidly obese patients who failed to lose weight by conservative methods. In the past decade, laparoscopic adjustable gastric banding (LABG) has become the procedure of choice for most of these patients. More than $50 \%$ to $80 \%$ of LAGBs are performed in women, of which about one-half are of reproductive age. Therefore, pregnancy with the LAGB device in situ is common. ${ }^{1,2}$ Although LABGs are generally safe and well tolerated during pregnancy, they may cause intractable nausea and vomiting, which are resolved with deflation of the silicon band. ${ }^{3}$ Other complications associated with the LABG procedure, such as erosion of the band into the stomach, leakage, and other problems related to the access port, are generally rare. ${ }^{4}$ In this case report, we describe an unusual complication of LABG in pregnancy, the entrapment of the adnexum by the connecting gastric band.

\section{CASE DESCRIPTION}

The patient is a 34-year-old woman, gravida 2, para 1, who underwent the LAGB procedure for morbid obesity (body mass index of $43 \mathrm{~kg} / \mathrm{m}^{2}$ ) 3 years prior to her current presentation. Her past surgical history was also significant for a cesarean section in her previous pregnancy. The index pregnancy was a singleton pregnancy achieved by in vitro fertilization treatment for male infertility. She was hospitalized twice for ovarian hyperstimulation syndrome and was treated by parenteral albumin and paracentesis of 
her ascites. Her prepregnancy body mass index was 32.3 $\mathrm{kg} / \mathrm{m}^{2}$. The patient presented at 14 weeks' gestation with severe left lower quadrant abdominal pain and nausea. Her pain started a few hours earlier and woke her from sleep. On physical examination, her blood pressure was $114 / 73 \mathrm{~mm} \mathrm{Hg}$, and her heart rate was 82 beats $/ \mathrm{min}$. She was afebrile. On abdominal examination, the lower abdomen, particularly on the left side, was tender to palpation. The abdominal palpation also elicited rebound tenderness and guarding. Bowel sounds were detected on auscultation. On pelvic bimanual examination, the uterus was enlarged to 14 weeks' gestation and the adnexa were both enlarged. The left adnexum was extremely tender to palpation and was located in the mid abdomen. The cervix was not dilated or effaced.

Sonographic evaluation using vaginal and abdominal probes demonstrated a viable singleton fetus with a biometry consistent with the gestational age. The right ovary contained numerous cystic structures consistent with ovarian stimulation and was enlarged to $5.4 \times 6.8 \mathrm{~cm}$ in diameter. The left ovary had a similar appearance and was enlarged to $8.5 \times 5.6 \mathrm{~cm}$ in diameter. The left ovary was located in the mid abdomen, and the sonographer's impression was of stromal edema. On Doppler flow examination, no blood flow was demonstrated to the left ovary. A small amount of free pelvic fluid was seen.

Laboratory test results revealed the complete blood cell count, liver and renal function, and coagulation tests were within normal limits. C-reactive protein was mildly elevated to $24.9 \mathrm{mg} / \mathrm{L}$ (normal upper limit is $5 \mathrm{mg} / \mathrm{L}$ ).

Based on the presumed diagnosis of left adnexal torsion, the patient was prepared for an urgent laparoscopic procedure. Because of the size of the gravid uterus, laparoscopy was performed with placement of the Veress needle in the left upper quadrant (Palmer's point) well above the uterine fundus. Following creation of the pneumoperitoneum, a 5-mm port was inserted in the left upper quadrant, followed by two 5-mm accessory ports inserted under direct visualization. On laparoscopy, the left adnexum was found to be entrapped by the connecting tube of the LAGB device. The left adnexum was released using an atraumatic grasper and regained its normal color and appearance (Figure 1). The postoperative course was uneventful, and the patient was discharged home 2 days later. The pregnancy proceeded without further complication, and the patient delivered on term by repeat cesarean section.

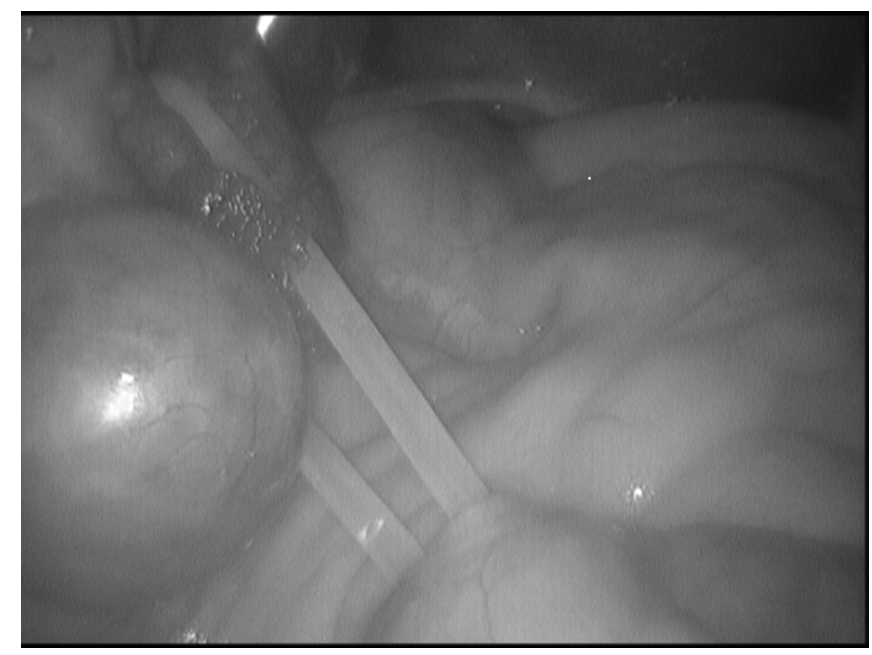

Figure 1. Laparoscopic picture of the gastric band wrapped around the left adnexum.

\section{DISCUSSION}

LABGs are commonly performed in women of reproductive age, ${ }^{1}$ making this procedure a relatively common feature in pregnancy. Most pregnancies with LABGs are uneventful, although intractable nausea and vomiting may be present due to the increased intra-abdominal pressure associated with pregnancy. ${ }^{3}$ Other less common complications of LABGs in pregnancy may include leakage and disconnection of the tube ${ }^{4}$ and, rarely, strangulation of the upper jejunum through an internal hernia. 5 In this case report, we present an unusual complication of the LABG in a 14 weeks' gestation pregnancy, where the gastric band entrapped the left adnexum, causing a decrease to its blood supply, adnexal ischemia, and a clinical and sonographic picture resembling adnexal torsion. This complication was probably enabled by the increased size and multicystic appearance of the adnexum, which is a common sequela of in vitro fertilization treatment. ${ }^{6}$

Laparoscopy has been used increasingly in pregnancy for management of gynecologic conditions such as adnexal torsion and ovarian cysts and nongynecologic conditions such as acute appendicitis. ${ }^{7,8}$ Naturally, special considerations are required when performing laparoscopy in pregnancy, such as safe abdominal entry, patient positioning, port placement, insufflation pressure, and postoperative monitoring and treatment of the patient and the fetus (including considering tocolytics and venous thromboembolic prophylaxis). ${ }^{9}$ In the current case, the laparoscopic approach enabled diagnosis and release of the 
torsion without complications. The abdominal entry was performed using the close approach with the Veress needle placed in the left upper quadrant. Other available techniques for abdominal entry in the second and third trimesters are the open approach and the use of optical blunt trocars. The different parameters such as surgeons' experience and patients' characteristics would determine the preferable technique for abdominal entry in each case.

Although, to our knowledge, this is the first case report to describe this complication in the literature, the increasing use of LABG in women of reproductive age may bring on similar cases in the future. We suggest awareness of this condition in gravid women with a history of LABG procedure and acute abdominal pain.

\section{References:}

1. Ducarme G, Revaux A, Luton D. Bariatric surgery and obstetrics [in French]. J Gynecol Obstet Biol Reprod. 2009;38(2):107116.

2. Shekelle PG, Newberry S, Maglione M, et al. Bariatric surgery in women of reproductive age: special concerns for pregnancy. Evid Rep Technol Assess (Full Rep). 2008;(169):1-51.
3. Guelinckx I, Devlieger R, Vansant G. Reproductive outcome after bariatric surgery: a critical review. Hum Reprod Update. 2009;15(2):189-201.

4. Haward RN, Brown WA, O'Brien PE. Does pregnancy increase the need for revisional surgery after laparoscopic adjustable gastric banding? Obes Surg. 2011;21(9):1362-1369.

5. Wang CB, Hsieh CC, Chen CH, Lin YH, Lee CY, Tseng CJ. Strangulation of upper jejunum in subsequent pregnancy following gastric bypass surgery. Taiwan J Obstet Gynecol. 2007;46(3): 267-271.

6. Smorgick N, Pansky M, Feingold M, Herman A, Halperin R, Maymon R. The clinical characteristics and sonographic findings of maternal ovarian torsion in pregnancy. Fertil Steril. 2009; 92(6):1983-1987.

7. Al-Fozan H, Tulandi T. Safety and risks of laparoscopy in pregnancy. Curr Opin Obstet Gynecol. 2002;14(4):375-379.

8. Tazuke SI, Nezhat FR, Nezhat CH, Seidman DS, Phillips DR, Nezhat CR. Laparoscopic management of pelvic pathology during pregnancy. J Am Assoc Gynecol Laparosc. 1997;4(5):605608.

9. Nezhat FR, Tazuke S, Nezhat CH, Seidman DS, Phillips DR, Nezhat CR. Laparoscopy during pregnancy: a literature review. JSLS. 1997;1(1):17-27. 